\title{
Le torri costiere del Regno di Sardegna: costruzione, danni e restauri
}

\section{Daniele Vacca}

Istituto di Storia dell’Europa Mediterranea del CNR, Cagliari, Italia, vaccadaniele@ hotmail.it

\begin{abstract}
This works has the goal to locate the organisation and the evolution of the systems of costal towers in the Kingdom of Sardinia, integrated in the defence organization of the Spanish Kingdom. The development of the defence system went through huge difficulties, including financial problems and from the enemy attacks. This required many times, some work on the towers, to keep them in a good state, or to solve other kind of problems, related to the use of bad materials and other illegitimate and illegal actions. Some natural causes also where the reason for the deterioration of the defence and military places, like natural causes or disasters, like for example, the earthquake in the 1616; a very rare event for the island, that started in the coastline of Capo Carbonara and caused a lot of damages in 8 towers; reason why the administration had to find a quick solution.
\end{abstract}

Keywords: Edad Moderna, torres costeras, Cerdeña, terremoto

\section{Introduzione}

Lo sviluppo del sistema di difesa costiero del Regno di Sardegna fu istituito, ufficialmente, alla fine di settembre del 1587 , dopo la ratifica da parte di Filippo II del documento contenente le richieste stamentarie o dei tre bracci dei Parlamenti sardi. Occorre dire, in realtà, che già diversi anni prima di questa data il sovrano aveva cercato di premunirsi, inviando ingegneri ed esperti di strategia militare per cercare di capire quale fosse lo stato delle difese dell'Isola e in quali punti sarebbe stato opportuno migliorarle e potenziarle.

Con l'abbandono della politica interventista di Carlo I, il successivo depotenziamento militare e la conseguente perdita dei baluardi difensivi del nord Africa, l'Isola diventò, con la Sicilia, una vera e propria testa di ponte verso il mondo islamico; peraltro, quest'ultima era decisamente più dotata di opere di opere difensive, tanto da essere definita la fortezza del Mediterraneo.
L'intento di questo intervento è quello di mettere in evidenza come le coste del Regno di Sardegna siano state modificate nel corso del tempo, cosa che abbiamo voluto mostrare anche graficamente, per dare un'idea più chiara dello sviluppo della difesa nel Regno di Sardegna tra la fine del Cinquescento e i primi decenni del Seicento.

Il primo studio relativo alle difese costiere dell'Isola sono del 1572, anno in cui il sovrano inviò il capitano d'Iglesias Marco Antonio Camos in giro per la Sardegna, questi aveva il compito di stilare una relazione sullo stato delle difese ma, soprattutto, aveva il compito di indicare qui siti d'importanza strategica, da difendere con la costruzione di torri di avvistamento, sensillas o di difesa de armas; nella stessa egli indicava i punti da cui trarre i materiali, per la loro costruzione, il numero dei soldati per ogni torre e su chi dovevano ricadere le spese per il loro approvvigionamento. Come si 
evince chiaramente ciò avvenne qualche anno prima della definitiva perdita delle fortezze di $\mathrm{La}$ Goleta e di Tunisi. A quell'epoca in Sardegna, oltre che le città costiere fortificate, come Cagliari, Iglesias, Bosa, Oristano, Alghero, Sassari e Castellaragonese, si potevano contare solo pochissime torri litoranee, realizzate in parte nel periodo catalano-aragonese, in parte nella prima metà del Cinquecento.

Lo sviluppo del sistema di difesa costiero nel Regno di Sardegna è stato tratto dalle diverse relazioni che i vari visitatori e/o uomini d'arme realizzarono, in base a precisi ordini del re Filippo II; ciò consentì al sovrano di avere un quadro chiaro sullo stato della difesa costiera dell'Isola e di intervenire secondo le necessità, per la difesa di luoghi di particolare interesse strategico o per difendere le remunerative attività economiche presenti lungo le coste: peschiere, saline, tonnare, coralline. Le relazioni a cui abbiamo fatto riferimento sono le Fig. 1, 2 3 e 4 .

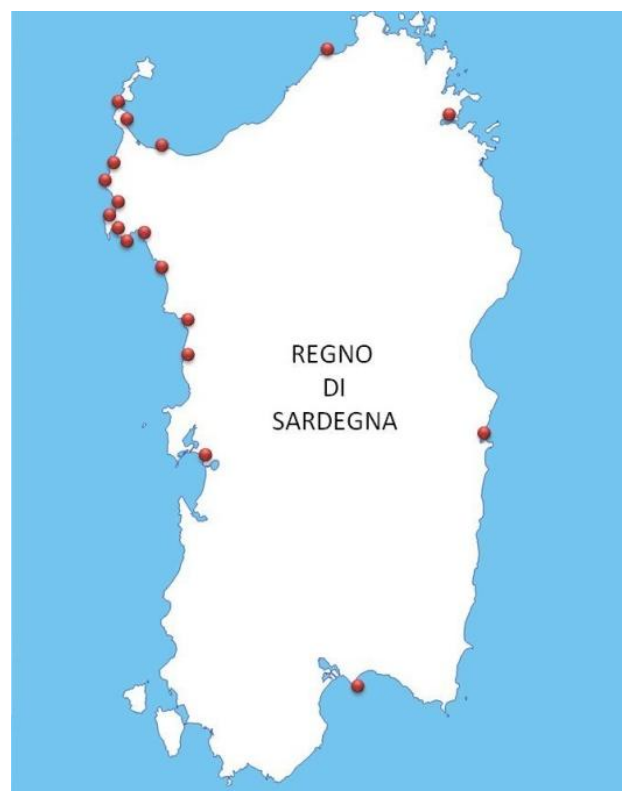

Fig.1- 1572. Le 18 torri costiere indicate dal capitano di Iglesias Marco Antonio Camos.

Dalle carte delle torri, realizzate in base alle diverse relazioni, si potrà notare come nel periodo precedente alla perdita definitiva dei presidi nord-africani, da parte della Corona di
Spagna, la Sardegna fosse scarsamente e malamente fortificata, ma pian piano, in particolare dopo il 1574, anno della caduta delle fortezze di La Goletta e di Tunisi, lo sviluppo del sistema di difesa statico subì una netta accelerazione. In realtà, a ben vedere, tale sviluppo era già stato avviato ancor prima che iniziassero i lavori del Parlamento Moncada del 1583, poi ratificati, come detto in precedenza, il 29 settembre 1587. Dagli inizi degli anni Ottanta, fino a circa il $1610 / 1620$, furono realizzate quasi 80 torri; circa 50 se si parte proprio dall'istituzione dell'ufficio della Administración del real. Certo l'istituzione del sistema di difesa costiero, definito sistema di difesa statico, non determinò la fine delle incursioni, ma questa fitta rete difensiva fu certamente un valido deterrente contro gli assalti nemici. Un altro aspetto che ci preme mettere in evidenza è il fatto che, ieri, così come oggi, vi fosse un vero e proprio malcostume nella realizzazione di queste opere 'pubbliche', o meglio, di pubblica utilità, necessarie, non solo, per la sopravvivenza fisica delle persone, ma anche per quella sociale; ovvero la sopravvivenza di tutte quelle importantissime attività economiche che si svilupparono nelle coste nel corso di svariati secoli, fatto di abusi, malversazioni e ruberie d'ogni genere che non fecero altro che rallentare e mettere a rischio l'esistenza stessa del sistema di difesa del regno. Lo sperpero di denaro dovuto all'uso di materiali inadeguati o di scarsa qualità, le malversazioni, spesso entrambe le due cose e svariati altri comportamenti che spesso sfociavano nell'illegalità, costrinsero l'Amministrazione delle torri ad intervenire più per il restauro delle stesse, sebbene fossero state terminate soltanto qualche anno prima, che per edificarne di nuove. Come si può dedurre facilmente, gli interventi per i cosiddetti adobs e l'utilizzo dei proventi del dret del real, non davano la possibilità di edificare nuove torri, come si sarebbe voluto e forse potuto. Spesso questi abusi furono scoperti e denunciati, e in diverse occasioni gli autori di tali comportamenti criminali furono costretti a rimborsare il maltolto con giornate di lavoro gratuite per la comunità o l'amministrazione delle torri, ma il più delle volte si spalancarono 
agli stessi le porte del carcere. Dai documenti presi in esame si evince che a pagare erano più frequentemente coloro che avrebbero dovuto controllare che i lavori fossero stati svolti ' $a b$ tota perfectiò', secondo quanto disposto dal memorial, che può tranquillamente essere paragonato a quello che oggi viene stabilito nelle gare d'appalto, il computo metrico estimativo. $\mathrm{Nel}$ caso in cui i lavori non fossero stati realizzati secondo quanto stabilito, era proprio lui, come spesso accadde, a risponderne personalmente, con i propri beni o addirittura con il carcere: 'se troba mala fabrica axi de calsina, arena, pedra y cantons et altres $y$ aquelles no estiguen adobades ab tota perfectiò conforme dit memorial que ell pagarà en sos bens y persona tot lo dany que se causarà de qualsevol manera que sia per dita mala fabrica per lo que ne obligase persona y bens'.

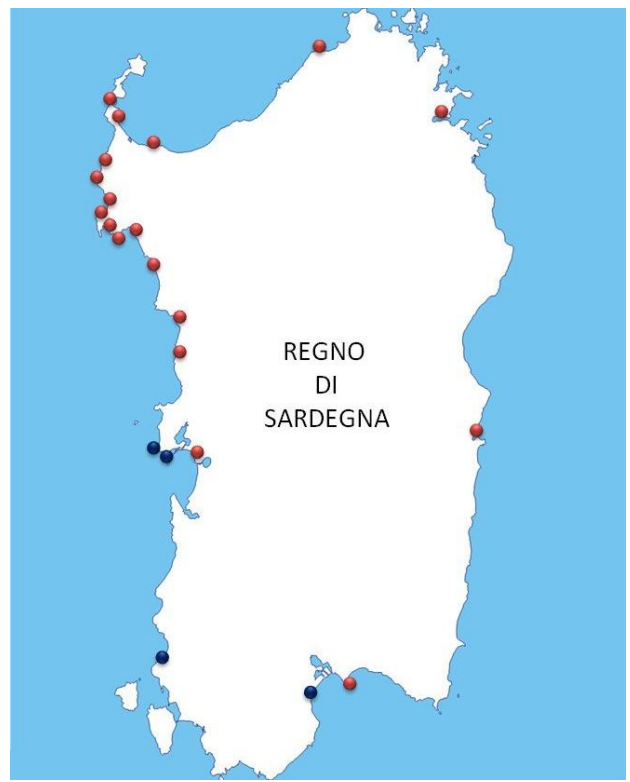

Fig.2- 1578. Le 22 torri costiere indicate dal viceré Miguel de Moncada.

Uno degli episodi presi in considerazione riguarda $i$ fatti e le vicende riguardanti la realizzazione della torre di San Luigi nell'Isola di Serpentara, ultimata nel 1608. La spesa complessiva per l'edificazione di questa importante torre costiera, fu di 9969 lliure, come è testimoniato in un documento dell'Ufficio della Amministrazione delle torri dell'anno successivo.

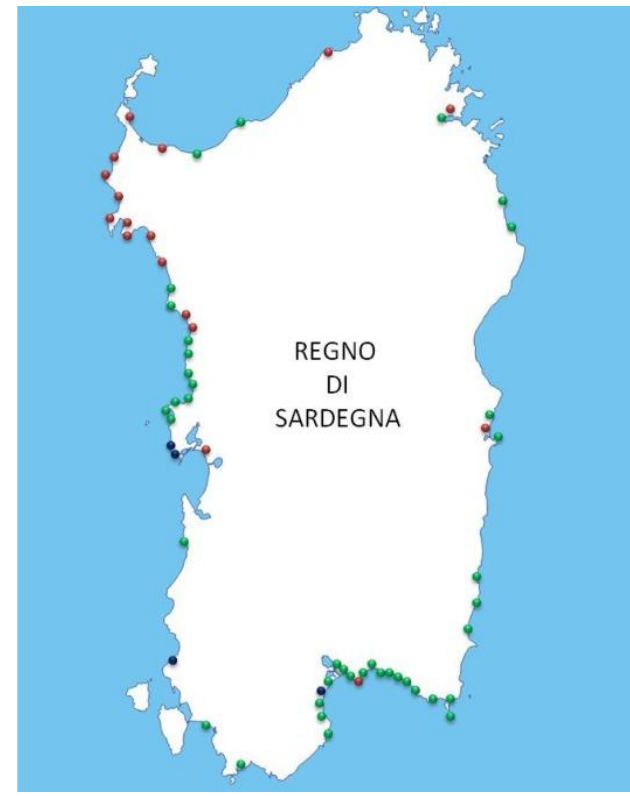

Fig.3- 1584/1587. Le torri costiere indicate in una. nuova relazione del viceré Miguel de Moncada.

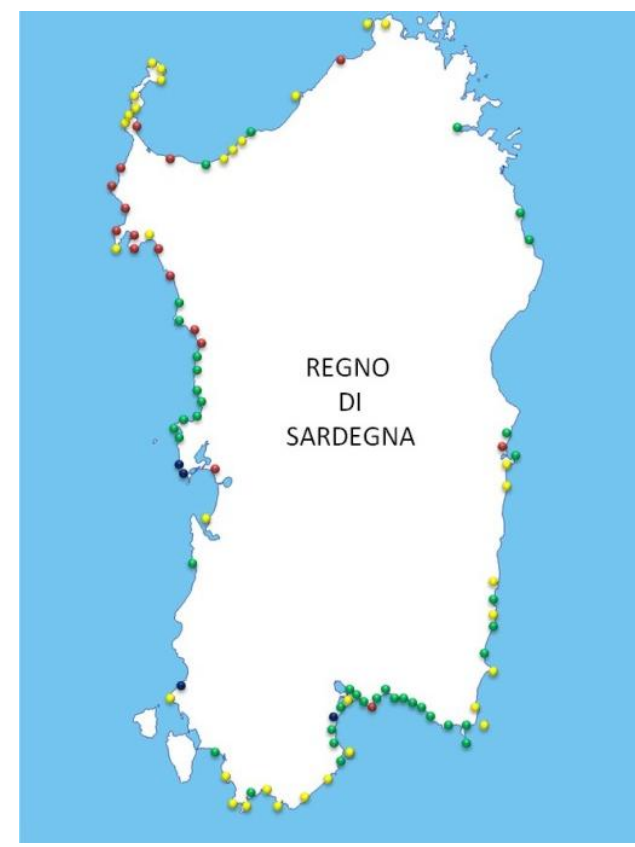

Fig.4- 1639. Le torri costiere indicate nell'opera di Francesco Vico. 
Sappiamo che nel gennaio 1610 l'alcaide della medesima torre, Hieroni Ollosa, si trovava in carcere, e si trovava in stato di detenzione da circa tre mesi, visto che gli furono decurtati tre mesi di paga dal suo salario, per pagare un altro soldato che fu incaricato della difesa di quella importante torre. In altri frangenti, le pene potevano colpire $\mathrm{i}$ beni o il patrimonio, per esempio, come accadde ai due mestres: 'Mestre Julià Taris y mestre Sissinni Setxi, picapedrers' che furono obbligati a produrre a loro spese, '.. que dins termini de un mes del dia present en avant contador daran y posaran a ses despesses tota la calsina del forn que faran pastada ab aygua dolça y arena dolça en la fornaçe a prop de la torre de la de Sancto Macari a rahò deu lliures lo cop de dita calsina pastada'. I casi di cui sopra, non furono certamente sporadici, si verificavano di frequente e l'ufficio dell'amministrazione doveva chiaramente verificare, con i propri ufficiali, al che tutti i lavori fossero tutti realizzati 'a regola d'arte'.

Tali malversazioni e abusi furono posti in evidenza, in particolare, da Martin Carrillo con la sua 'visita' del 1611, da cui scaturirono le sue inchieste, che continuarono a lasciare tracce anche qualche anno dopo la sua partenza dall'Isola, le citano come pratiche comuni, e in tali illecite pratiche furono coinvolti anche $\mathrm{i}$ più alti ufficiali regi, senza che però si fosse riusciti seriamente a porre un freno a tali abusi; tanto che gli effetti si videro nei parlamenti successivi.

Questi comportamenti furono tra i motivi principali non solo del rallentamento, ma probabilmente anche del mancato sviluppo, per cui le opere realizzate nel Regno di Sardegna, dopo una partenza ritardata non furono così numerose come ci si sarebbe attesi e come sarebbe stato opportuno fare, vista anche la posizione dell'Isola. Con una più oculata e trasparente gestione si sarebbero potuti limitare ancor più i danni, le sofferenze e le deportazioni che tali terribili assalti determinarono; se si fosse lavorato con onestà e criterio, limitando tutti questi inutili sprechi di denaro, probabilmente, si sarebbe potuto creare un sistema di difesa più valido ed efficiente di quello che fu effettivamente realizzato.
Oltre agli esempi di illeciti da parte di diversi personaggi, di cui abbiamo appena detto, che con i loro comportamenti disonesti causarono un grave danno alle casse della amministrazione, occorre anche mettere in rilievo il fatto che molti interventi si resero necessari anche, ma non solo, per riparare i danni causati dagli innumerevoli e violenti assalti dei corsari o della pirateria turca, come avvenne nelle due torri di Longosardo e di La Testa nel Judicado di Gemini-Gallura, presso l'attuale Santa Teresa Gallura. Le due torri furono completamente distrutte, una era stata appena conclusa, mentre per ultimare l'altra mancavano solo pochi giorni di lavoro, in questo caso fu necessario intervenire immediatamente per la ricostruzione, vista anche l'importanza della zona da difendere. Ma si dovette intervenire anche, in alcuni altri casi, per ovviare ad incredibili e straordinari eventi atmosferici, delle vere e proprie calamità naturali, quali fulmini e lampi. Nel 1607, infatti, dopo appena due anni dalla sua edificazione, la torre di Cala di Piombo, ubicata lungo la costa sudoccidentale dell'Isola, fu colpita da un fulmine che causò gravissimi danni. ' la torre del Cap del Plom la qual segons se diu derrota part de aquella lo trò y lamp que caygue...' I lavori di restauro furono assegnati all'impresario Pere Porta e al maestro scalpellino Miquel Pinna che vinse la gara d'appalto; dal documento si evince anche che 'lo adob y reparo de dita de dita torre del Cap del Plom en dit preu de dits tres milia lliures'; vale a dire, che la spesa totale per gli interventi necessari fu di 3000 lliures, ovvero poco meno di un terzo del denaro necessario per la realizzazione della torre di San Luigi nell'Isola di Serpentara.

L'evento più clamoroso ed inusuale non fu quest'ultimo, ovvero la caduta di un tuono e di un fulmine, ma qualcosa di ancor più inimmaginabile ed inusuale per la nostra Isola, accadde certamente a poche miglia di distanza da Capo Carbonara, zona posta nella parte sudorientale della Sardegna, fu in quel luogo infatti che il 4 giugno 1616 vi fu un forte terremoto che causò numerosi danni a ben otto torri lungo la fascia costiera che va da Cala Pira alla torre di Cala Regina. Anche in questo caso i costi per la ristrutturazione delle otto torri del sistema di 
difesa costiero furono ingenti, l'appalto infatti fu assegnato per un'offerta di 3.950 lliures ai mestres picapedrers Sissinni Setxi, Sebastià Cau e Pere Joan Pintus.

\section{Conclusioni}

Potremmo fare numerosi altri esempi, per dare sostanza a questa nostra considerazione, e tutti ci porterebbero alla medesima conclusione; i restauri delle torri costiere, e delle strutture fortificate in genere, erano indubbiamente indispensabili e certamente inevitabili, dopo un certo numero di anni, ma è pur vero che in passato, così come purtroppo accade anche oggi, i maggiori interventi e le maggiori spese siano state determinate per la maggior parte da comportamenti negligenti; spesso dovuti all'incapacità di alcuni personaggi e alla loro scarsa preparazione, altrettanto spesso all'incuria, ma il più delle volte le cause sono da ricercarsi nei comportamenti illeciti di persone che per arricchirsi ad ogni costo commisero diversi abusi e numerosi reati.

Alcuni si limitarono ad utilizzare materiali scadenti, altri mestres picapedrers ad esempio, realizzarono e fecero realizzare impasti con l'acqua di mare, molto più economica, ma che esponeva le strutture ad una maggiore erosione, altri rubando e rivendendo $\mathrm{i}$ materiali e i rifornimenti provenienti dalla stessa amministrazione, o commettendo diversi reati contro il patrimonio, tutto ciò determinò enormi difficoltà alla stessa Real Administración precludendogli in diversi casi la possibilità di realizzare nuove opere di difesa e, nel contempo, di rendere sempre più sicuro l'intero sviluppo costiero della nostra Isola.

\section{References}

Anatra, B. (1987). La Sardegna dall'unificazione aragonese ai Savoia, Torino, pp. 288-290.

Mattone, A. (1989). "Le istituzioni militari”, Storia dei Sardi e della Sardegna, III, Milano, p. 68.

Cámara A. (1998). "Las fortificaciones y la defensa del Mediterráneo", in Felipe II y el Mediterráneo. La monarquía y los reinos. Convegno Internazionale, Barcellona, IV, a cura di Ernest Belenguer Cebrià, Madrid, 1999, p. 359.

Cámara A. (1990). "Las torres del litoral en el reinado de Felipe II: una arquitectura para la defensa del territorio", Espacio, Tiempo y Forma, 3, pp. 55-86 e 4, 1991, pp. 53-94.

Castelli P. (1984). "La progettazione del sistema territoriale di difesa", Atti del Convegno Nazionale Arte e Cultura del '600 e del'700 in Sardegna, (Cagliari-Sassari, 2-5 maggio 1983), Tatiana K. Kirova (a cura di), Napoli, pp. 45-46 e 48.

Dexart J. (1641). Capitula sive Acta Curiarum Regni Sardiniae, sub invictissimo Coronae Aragonum imperium concordi trium brachiorum aut solius militaris voto exorata, veteri ex codice et actis novissimo rum proprias in sede ac materias coacta, lib. I, Tit. IIII, de Capitulis Carta Localis et aliis Constitututionibus, Cagliari, pp. 146-175.

Favarò V. (2004). "La Sicilia fortezza del Mediterraneo”, in Mediterranea. Ricerche storiche, n. 1, giugno.

Mele G. (2000). Torri e cannoni. La difesa costiera in Sardegna nell'età moderna, Sassari, pp. 54-64 e $125-136$.

Mele G. (2008). "Torri o galere? Il problema della difesa costiera in Sardegna tra il XVI e il XVIII secolo", Atti del Convegno Internazionale di Studi Contra Moros y Turcos, Politiche e sistemi di difesa degli Stati mediterranei della Corona di Spagna in Età Moderna, (Villasimius-Baunei, 20-24 settembre 2005), Dolianova (CA), pp. 197-207.

Mele G. (2006). "Raccolta di documenti editi e inediti per la Storia della Sardegna", vol. 7, Documenti sulla difesa militare della Sardegna in età spagnola, Sassari, pp. 244-251.

Mele, G. (2005). «...en gran perill de moros i de enemichs...»: intenti e operatività nella difesa costiera del Cinquecento", Atti del Convegno Internazionale di Studi Contra Moros y Turcos, Politiche e sistemi di difesa degli Stati mediterranei della Corona di Spagna in Età Moderna, VillasimiusBaunei, 20-24 settembre, pp. 139-153. 
Murgia G. (2008). "Presenza corsara nel Mediterraneo Occidentale e problemi di difesa nel Regno di Sardegna (secoli XVI-XVII)", Atti del Convegno Internazionale di Studi Contra Moros y Turcos, Politiche e sistemi di difesa degli Stati mediterranei della Corona di Spagna in Età Moderna, (Villasimius-Baunei, 20-24 settembre 2005), Dolianova (CA), p. 183.

Pillosu E. (1961). "Un inedito rapporto cinquecentesco sulla difesa costiera della Sardegna di Marco Antonio Camos", Nuovo Bollettino Bibliografico Sardo, IV n. 21, 22, 23 e 24, 1959; V. n. 25, Cagliari, pp. 13, 14 e 32.

Plaisant, M.L. (1969). "Martin Carrillo e le sue relazioni sulle condizioni della Sardegna”, Studi Sardi, vol XXI, Sassari, pp. 175-262.

Russo F. (1991). "La difesa costiera del Regno di Sardegna dal XVI al XIX secolo”, Stato Maggiore dell'Esercito. Ufficio Storico, Roma, p. 69-73.

Vacca D. (2009). "Le torri litoranee della costa Sud-Occidentale della Sardegna e la difesa delle isole minori dagli attacchi dei corsari barbareschi”, Sardegna e Mediterraneo tra Medioevo ed Età Moderna. Studi in onore di Francesco Cesare Casula, a cura di M. G. Meloni e O. Schena, Genova, p. 435.

Vacca D. (2011). Restauro delle torri della Sardegna costa Sud-Orientale danneggiate dal terremoto del 1616, pubblicazione on-line in http://www.carlodelfinoeditore.it/public/docs/vaccaterremoto.pdf , Sassari.

Viganò, M. (2004). «El Fratìn mi ynginiero». I Paleari Fratino di Morcote, ingegneri militari ticinesi in Spagna (XVI-XVII secolo), Bellinzona, pp. 206-207.

Alberti, O.P. (1970). "Le carte della Sardegna di Rocco Cappellino", Nuovo Bollettino Bibliografico Sardo, XII, n. 72, pp. 3-7, Cagliari, p. 6. 